\title{
Caracterización de la roca soporte de los petroglifos de Campo Lameiro (Pontevedra)
}

\section{Characterization of the support rock of the petroglyphs of Campo Lameiro (Pontevedra)}

\author{
BENITA SILVA $^{1}$, PATRICIA SANMARTÍN ${ }^{{ }^{1}}$, BEATRIZ PRIETO ${ }^{1}$ \\ ${ }^{1}$ Departamento de Edafoloxía e Química Agrícola. Universidade de Santiago de Compostela (España). \\ * contacto: patricia.sanmartin@usc.es
}

https://doi.org/10.17979/cadlaxe.2019.41.0.5821

enviado: 1/1/2019 aceptado 1/11/2019

\begin{abstract}
Between 2005 and 2008 a research project about the deterioration causes of the petroglyphs of Campo Lameiro (Pontevedra) was developed. The substrate of the carvings was characterised as a two mica-granite, fine to medium grained, with granular allotriomorphic texture. Intense cracking is present in the rock at all levels, which is responsible of its high porosity and therefore its high capacity to absorb water. A dense network of diaclases was observed on site, however a specific study revealed that this does not involve a risk of massive loss of rock, which might affect the carved surfaces. The mineralogical and geochemical analysis showed that the granite was subjected to processes of hydrolysis and solubilisationprecipitation of iron, showing that water is the main deterioration agent in the lithic material.
\end{abstract}

Keywords: Heritage; Petroglyphs; Granite; Weathering; Preventive conservation; Archaeological park. 


\section{Resumen}

Entre los años 2005-2008 se llevó a cabo un proyecto de investigación para analizar las causas del deterioro de los petroglifos de Campo Lameiro (Pontevedra). La roca soporte de los grabados se caracterizó como un granito de dos micas de grano medio-fino y textura granuda equigranular alotriomorfa. Presenta una intensa fisuración a todos los niveles lo que le confiere una elevada porosidad y gran capacidad de absorción de agua. En campo se observa una densa red de diaclasas, sin embargo un estudio específico reveló que esto no supone riesgo de pérdida masiva de roca que pueda afectar a las superficies que poseen grabados. Los análisis mineralógicos y geoquímicos pusieron de manifiesto que el granito experimentó procesos de hidrólisis y de solubilización-precipitación del hierro, lo que indica que es el agua el principal agente de alteración en el material lítico.

Palabras clave: Patrimonio; Petroglifos; Granito; Meteorización; Conservación preventiva; Parque arqueológico. 


\section{INTRODUCCIÓN}

El municipio de Campo Lameiro es conocido por su riqueza en arte rupestre ya que concentra un gran número de petroglifos algunos de gran tamaño y diversidad figurativa (Figura 1). Este hecho, junto con circunstancias de tipo socioeconómico, han llevado a elegir este lugar, en concreto el área de Paredes (8॰31'49.6”W, 42³2'30.6”N, WGS84), para el emplazamiento del Parque Arqueológico del Arte Rupestre de Galicia ${ }^{1}$.

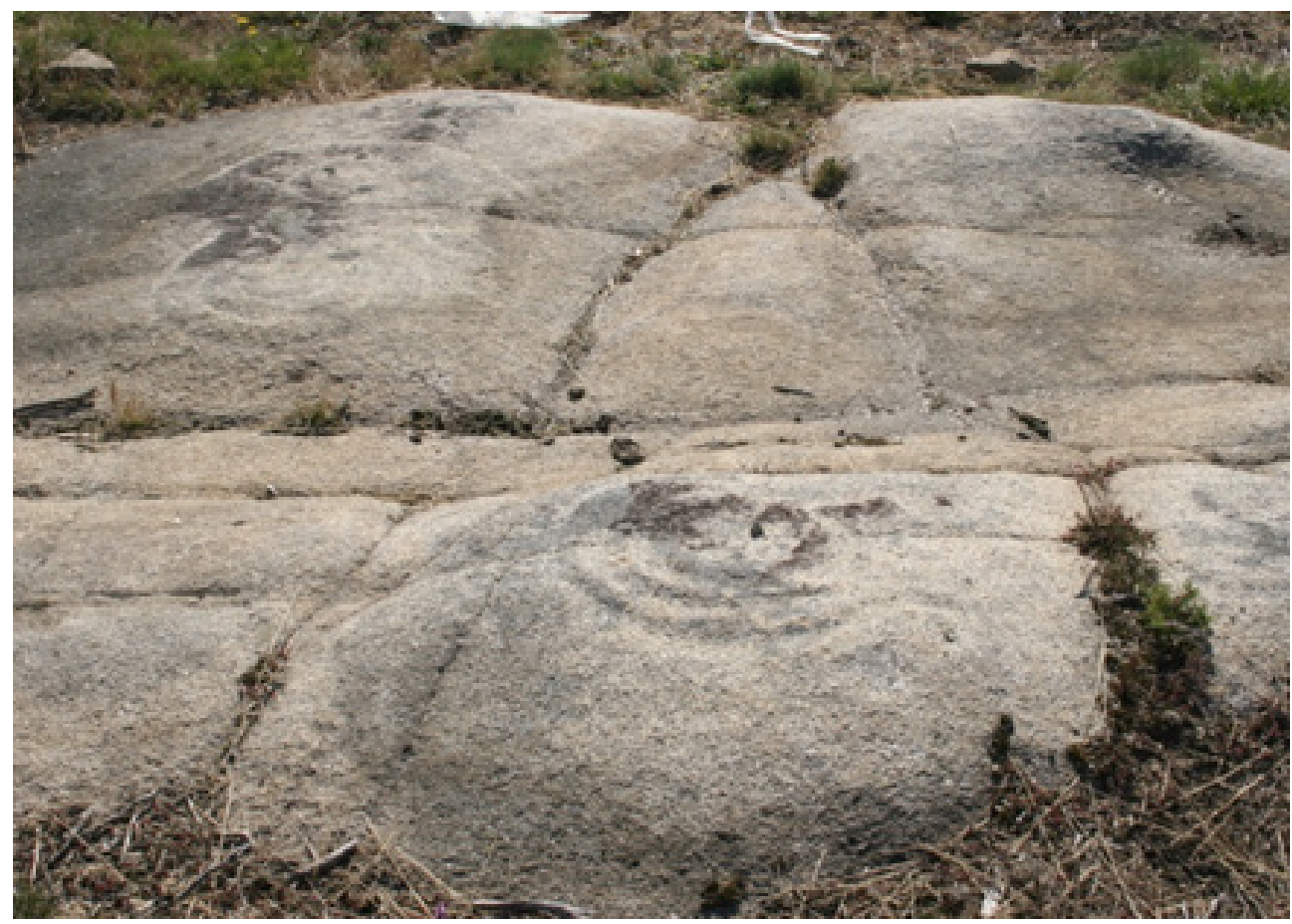

Fig. 1. Afloramiento granítico con grabados en el que se puede observar la densa red de diaclasas que atraviesa la roca.

A pesar del gran valor de estos elementos del patrimonio arqueológico como documentos para acercarnos a la cultura y costumbres de las comunidades que los crearon, su estado de conservación es muy deficiente. El deterioro de los grabados es notorio pues los surcos están muy erosionados, en ocasiones han sido repicados y pintados y en su gran mayoría están recubiertos con una pátina negra, circunstancias que dificultan enormemente la percepción de los motivos.

${ }^{1}$ Parque Arqueolóxico da Arte Rupestre Campo Lameiro. Recuperado de http://www.paar.es/?lang=en ${ }^{2}$ Silva, B. y colaboradores. Determinación de los factores de degradación de los petroglifos de Campo Lameiro. Diseño de medidas de conservación. Entidad financiadora: Xunta de Galicia (Ref. PGIDIT 05CCP20301PR). Periodo: Octubre 2005-Diciembre 2008. 
Esta situación suscitó la idea de desarrollar un proyecto de investigación financiado por la Xunta de Galicia que el Grupo de Estudios Medioambientales Aplicados al Patrimonio Natural y Cultural de la USC llevó a cabo entre los años 2005 y $2008^{2}$. El objetivo del estudio era analizar los factores causantes de la alteración de los petroglifos de Paredes para poder plantear las medidas adecuadas para frenar, o al menos paliar, su deterioro. Puesto que los grabados rupestres están realizados sobre afloramientos graníticos, es imprescindible en primer lugar conocer las características de la roca y el estado en que se encuentra y en segundo lugar analizar los factores ambientales y antrópicos que inciden en el deterioro de los grabados. En este artículo se presenta la primera parte del estudio, esto es la caracterización de la piedra y sus alteraciones.

\section{TOMA DE MUESTRAS Y ANÁLISIS REALIZADOS}

Se recogieron tres bloques de roca cerca de los petroglifos Laxe da Forneiriña I, Outeiro dos Cogoludos I y Outeiro das Ventaniñas ( $n^{\circ} 1,2$ y 3 respectivamente), y un cuarto bloque $\left(\mathrm{n}^{\circ} 4\right)$ cerca del edificio del centro de interpretación del parque arqueológico, en construcción en aquel momento. Estos bloques fueron extraídos de afloramientos rocosos de características similares y por tanto representativos de las rocas grabadas. Por otra parte, se tomaron 12 fragmentos de granito más pequeños en afloramientos cercanos a los petroglifos mencionados. A partir de los bloques se prepararon probetas cúbicas de $5 \mathrm{~cm}$ de lado para determinar las propiedades físicas de la roca y de todas las muestras se prepararon láminas delgadas de 50 micras de espesor para el estudio petro- gráfico y una parte se molió para los análisis de difracción de rayos $\mathrm{X}$ (DRX) y el estudio geoquímico.

Las láminas delgadas se estudiaron bajo microscopio óptico de luz transmitida para determinar la textura y la composición mineralógica de la roca, que se completó mediante análisis por DRX. La composición química se analizó mediante fluorescencia de rayos $\mathrm{X}(\mathrm{FRX}) \mathrm{y}$, por otra parte se determinó el pH de abrasión siguiendo el método de Grant (GRANT, 1969).

En cuanto a las propiedades físicas, se caracterizó el sistema poroso mediante la determinación de la densidad real (Dr), la densidad aparente (Da) y la porosidad abierta o accesible al agua (PA) y se determinaron las propiedades hídricas: contenido de agua en saturación (Ws), absorción libre de agua (Wa), y absorción de agua por capilaridad (Wc), todas ellas siguiendo las recomendaciones de la RILEM (R.I.L.E.M., 1980).

\section{RESULTADOS E INTERPRETACIÓN}

Las observaciones de campo permitieron comprobar que la roca del área de Paredes es un granito muy homogéneo en cuanto a granulometría y textura, característica positiva pues facilitó la toma de muestras al incrementar la representatividad de los resultados. La homogeneidad solo se rompe por la presencia de algunos filones pegmatíticos y por frecuentes venas pegmoaplíticas de cuarzo y moscovita que sobresalen en la superficie de los afloramientos por erosión diferencial por ser éstos los componentes más resistentes del granito. Llama la atención la coloración blanquecina de la superficie de muchos afloramientos y fragmentos sueltos, sin embargo cuando se parten se puede ver una franja subsuperficial enri- 
quecida en oxihidróxidos de hierro de color pardo-amarillento (Figura 2). Muchos otros afloramientos presentan manchas y bandas herrumbrosas pardo-amarillentas (Figura 2.b) que en ocasiones adquieren una colo- ración fuertemente rojiza, debido con toda probabilidad al efecto de los incendios ya que el calor origina la deshidratación de los oxihidróxidos de $\mathrm{Fe}$ convirtiéndose así en óxidos, de color rojo intenso.
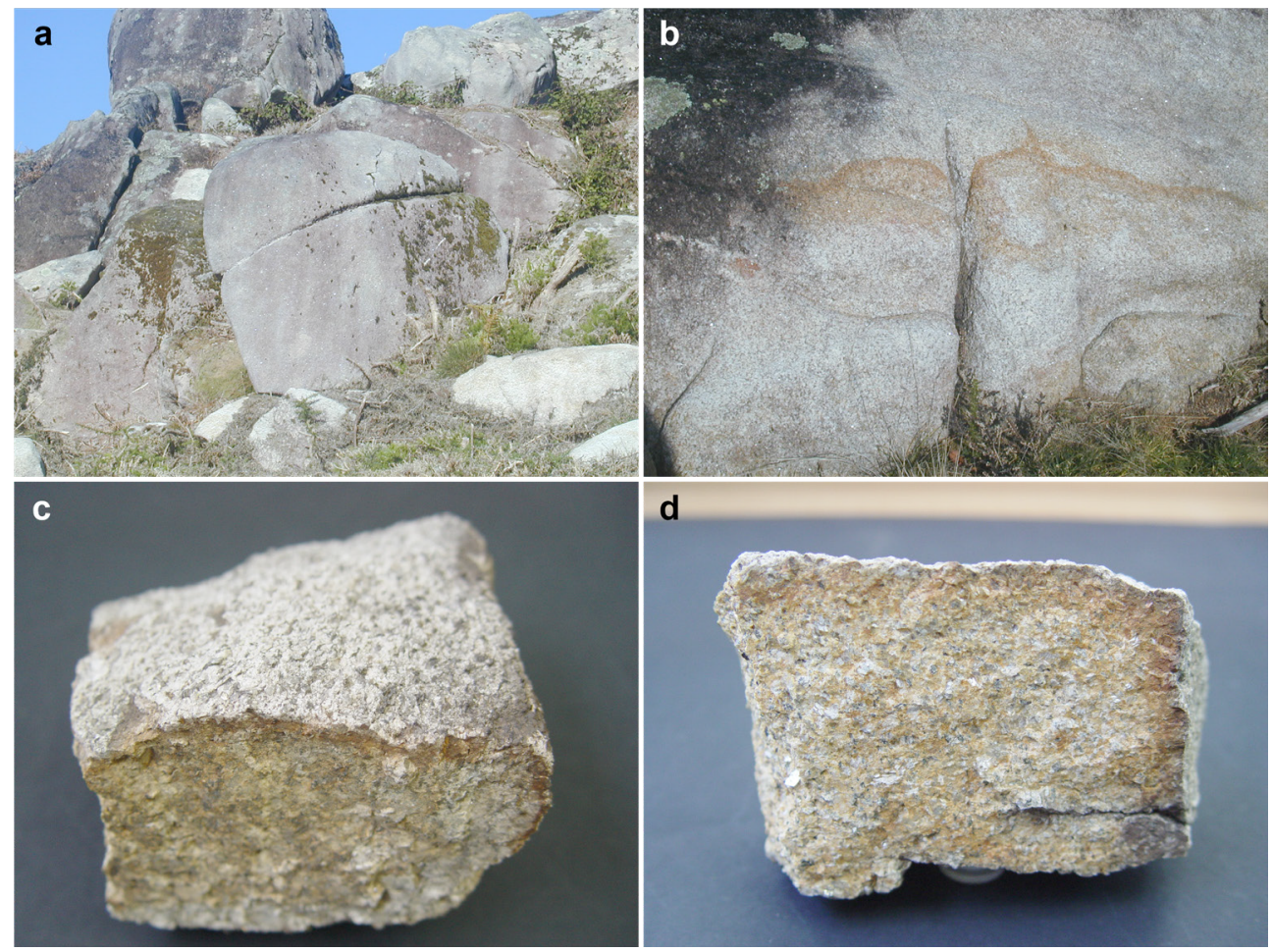

Fig. 2. (a): Afloramientos rocosos con diaclasas horizontales. (b): Bandas herrumbrosas de oxihidróxidos de hierro. (c y d): Fragmento rocoso con la superficie decolorada y un nivel subsuperficial enriquecido en oxihidróxidos de hierro.

Los afloramientos rocosos están muy fracturados (Figura 2.a) por lo que se realizó un estudio para evaluar si esto puede suponer algún riesgo de pérdida masiva de roca que ponga en peligro la pervivencia de los petroglifos. Se realizó en primer lugar la caracterización de la red de diaclasas, siguiendo las normas de la Sociedad Internacional de Mecánica de Rocas, identificándose dos familias de juntas principales de dirección noreste-suroeste y sureste-noroeste, sensiblemente ortogonales y muy verticales, y dos familias secundarias, además de otra serie de fracturas subhorizontales. Este conjunto de diaclasas verticales y subhorizontales divide el macizo en grandes bloques, en principio paralelepipédicos. Posteriormente se realizó un análisis de la estabilidad del macizo 
para evaluar si a través de las discontinuidades se podrían producir eventualmente deslizamientos de bloques de roca. Se concluyó que globalmente el macizo rocoso de Paredes presenta un buen comportamiento geomecánico y una escasa probabilidad de que se produzcan inestabilidades. Aún así, se evaluó específicamente la estabilidad de dos afloramientos con petroglifos: Forneiriña y Outeiro dos Cogoludos encontrándose que no existen riesgos de pérdida de masa que puedan afectar a las superficies que poseen grabados. Sin embargo se ha podido constatar que el agua que discurre por los petroglifos es canalizada según las familias de juntas identificadas marcando así las direcciones preferentes de meteorización química.

El estudio petrográfico (Figura 3) revela que se trata de un granito de dos micas de grano medio-fino, de textura granuda equigranular alotriomorfa. Sus componentes mayoritarios son: cuarzo (36\% en volumen), que presenta marcada extinción ondulante; feldespato potásico (18\%), el mineral de mayor tamaño que en general es microclina; plagioclasa (22\%), tipo oligoclasa-albita que se encuentra bastante fisurada y alterada presentando sericitación y transformación a minerales arcillosos en algunas zonas; biotita (7\%), de color pardo oscuro y fuerte pleocroísmo, en muchos casos transformada parcialmente (2 $\%$ aproximadamente) en clorita y con los bordes doblados; moscovita (13\%) que frecuentemente for-ma intercrecimientos con la biotita; y como accesorios (2\%) se identificaron apatito, circón, turmalina, rutilo y escasos granos de minerales opacos posiblemente óxidos de Fe.

La caracterización mineralógica del granito se completó con los análisis por DRX (Figura 4), técnica que permite reconocer además de los minerales más abundantes, otros que por ser escasos o de muy pequeño tamaño (como minerales arcillosos) no se pueden identificar con el estudio petrográfico, por eso es muy útil para detectar minerales secundarios que se producen por meteorización. El análisis de todas las muestras dio resultados muy similares, además de los minerales esenciales del granito (indicados en el estudio petrográfico) se identificaron dos minerales de neoformación: vermiculita que se forma por alteración de la biotita en medios ácidos, y caolinita producida en la hidrólisis de los feldespatos, sobre todo de las plagioclasas.

En algunas muestras se analizó separadamente la parte superficial decolorada y la franja herrumbrosa subsuperficial, hasta una profundidad de 1-1,5 cm. Se comprobó que en la primera los productos de meteorización (caolinita y vermiculita) están en menor proporción o incluso han desaparecido totalmente, lo que lleva a la interpretación de que en la parte superficial estos minerales arcillosos se han perdido por erosión mientras que en la franja subsuperficial se conservan debido a un cierto efecto cementante de los oxihidróxidos de hierro. 


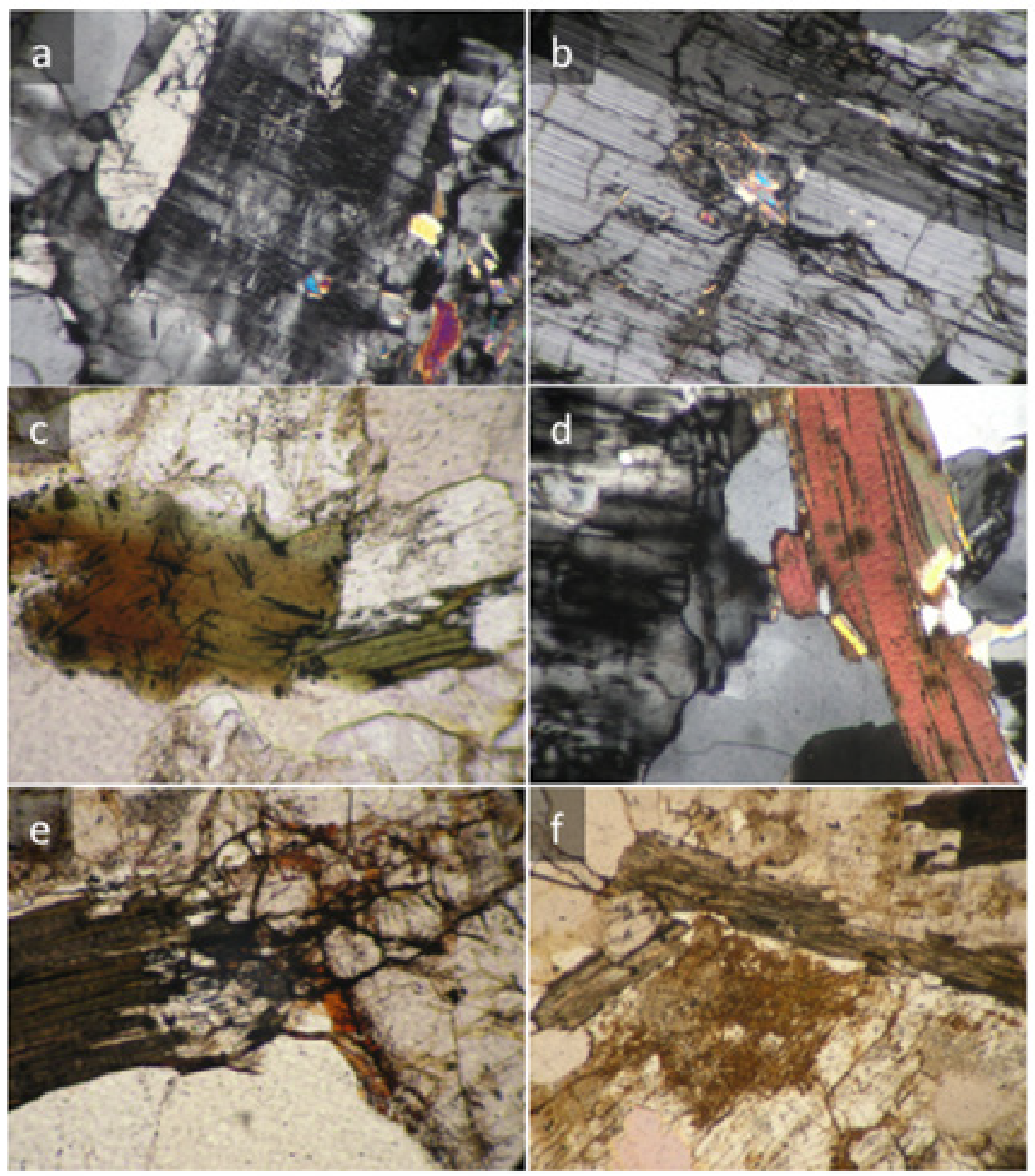

Fig. 3. (a): Cristal de microclina con la característica macla en enrejado. (b): Plagioclasa muy fracturada y sericitizada. (c): Biotita parcialmente cloritizada. (d): Cuarzo y biotita con inclusiones de circón. (e): cristal de biotita con la láminas abiertas del que salen oxihidróxidos de Fe que penetran en la fisuras de otros minerales. (f): Grano de plagioclasa parcialmente transformado en caolinita. (Fotos a, b y d con Nícoles cuzados; fotos c, d y f con Nícoles paralelos).

La composición química de diversas muestras analizadas se presenta en la Tabla
1. Los elementos más abundantes son el silicio ( $\mathrm{Si}$ ) y el aluminio ( $\mathrm{Al}$ ), que forman parte 
de todos los silicatos, y el potasio (K) ya que el feldespato potásico es un mineral mayoritario en estas rocas. El contenido de sodio (Na) supera ampliamente al de calcio $(\mathrm{Ca})$ lo que confirma que las plagioclasas son esencialmente albíticas, como se observó en el estudio petrográfico. El hierro (Fe) no es abundante pues es la biotita el único mineral en el granito que posee este elemento (además de algún accesorio); el titanio (Ti) suele ir asociado con el hierro (Fe). El fósforo (P) es un elemento que, aunque minoritario, es relativamente común en las rocas graníticas donde se encuentra frecuentemente en forma del mineral apatito.
Un parámetro muy útil para estimar el grado de meteorización de una roca es el pH de abrasión (Tabla 1) cuyo valor va descendiendo a medida que avanza el proceso (ROMERO et al., 1987). La mayoría de las muestras tienen un $\mathrm{pH}$ de abrasión próximo a 7,0 siendo los extremos el granito del bloque 4 con un $\mathrm{pH}$ de 8,0 y el de la muestra Cogoludos-3 con pH de 6,4. Teniendo en cuenta que los granitos sanos poseen $\mathrm{pH}$ de abrasión superiores a 9,0, estos datos indican que en términos generales el granito de Paredes presenta también desde el punto de vista geoquímico un grado de alteración notable.

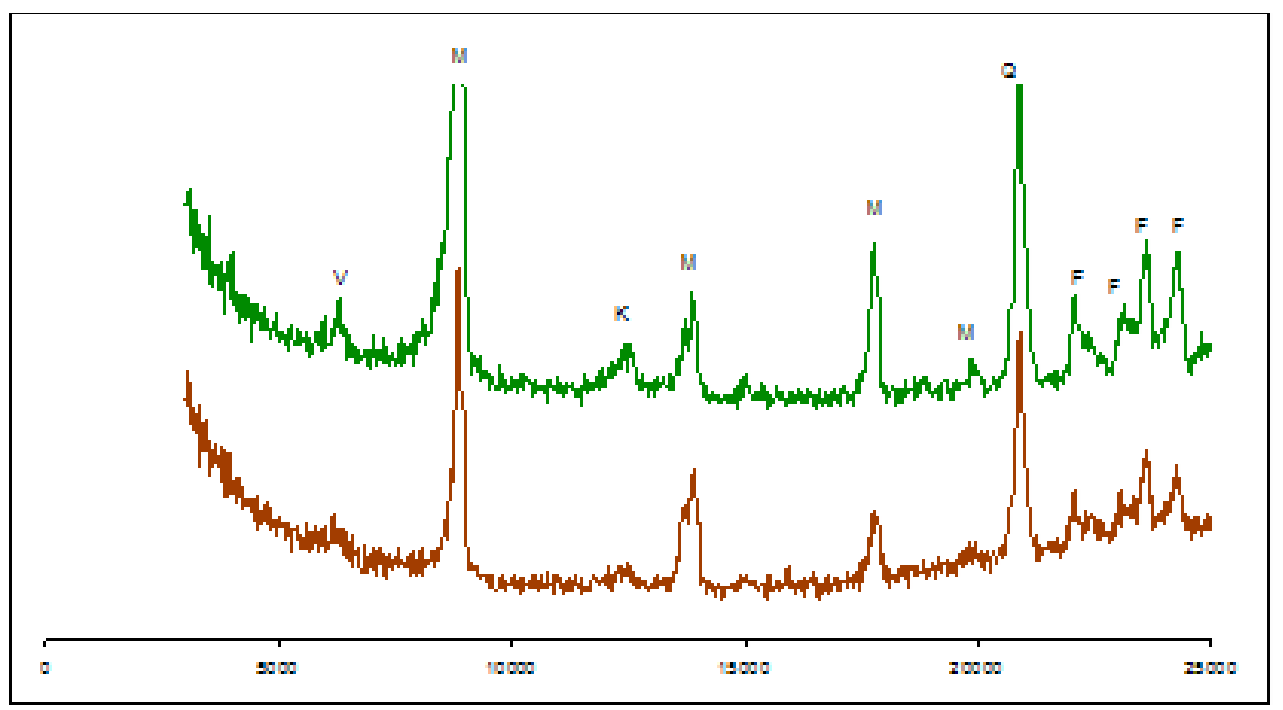

Fig. 4. Difractogramas de rayos X de dos submuestras de Forneiriña: arriba subsuperficial, abajo superficial. V: vermiculita; M: moscovita/biotita; K: caolinita; F: feldespato K/plagioclasa; Q: cuarzo. 


\begin{tabular}{|l|c|c|c|c|c|c|c|c|c|c|}
\hline & SiO2 & Al2O3 & Fe2O3 & MgO & CaO & Na2O & K2O & TiO2 & P2O5 & pH abrasión \\
\hline Cogoludos 3 & 74,19 & 15,08 & 1,20 & 0,22 & 0,31 & 2,77 & 5,72 & 0,17 & 0,21 & 6,43 \\
\hline Forneiriña 2 & 70,35 & 17,60 & 1,32 & 0,24 & 0,49 & 3,50 & 5,49 & 0,19 & 0,68 & 7,07 \\
\hline Ventaniñas 1 & 72,71 & 17,07 & 0,71 & 0,19 & 0,30 & 2,79 & 5,72 & 0,15 & 0,25 & 7,31 \\
\hline Bloque n'2 & 72,84 & 16,09 & 1,24 & 0,23 & 0,47 & 2,82 & 5,47 & 0,18 & 0,55 & 7,00 \\
\hline Bloque no3 & 70,89 & 16,90 & 1,37 & 0,25 & 0,50 & 3,74 & 5,57 & 0,17 & 0,54 & 7,41 \\
\hline Bloque no4 & 71,82 & 16,49 & 1,24 & 0,26 & 0,44 & 2,92 & 5,73 & 0,18 & 0,78 & 8,01 \\
\hline
\end{tabular}

Tabla 1. Composición química de las muestras de roca y pH de abrasión. Elementos mayoritarios expresados como $\%$ de óxidos.

\begin{tabular}{|l|c|c|c|}
\hline & Bloque $\mathbf{n}^{\mathbf{2}}$ & Bloque $\mathbf{n}^{\mathbf{3}}$ & Bloque $\mathbf{n}^{\mathbf{4}}$ \\
\hline $\mathbf{D a}\left(\mathbf{k g} / \mathbf{m}^{\mathbf{3}}\right)$ & $2413 \pm 8$ & $2393 \pm 21$ & $2529 \pm 12$ \\
\hline $\mathbf{D r}\left(\mathbf{k g} / \mathbf{m}^{3}\right)$ & $2641 \pm 3$ & $2641 \pm 4$ & $2640 \pm 5$ \\
\hline $\mathbf{P a}(\%)$ & $8.65 \pm 0.26$ & $9.39 \pm 0.85$ & $4.19 \pm 0.26$ \\
\hline Wa \% & $3.22 \pm 0.13$ & $3.60 \pm 0.36$ & $1.59 \pm 0.09$ \\
\hline Ws \% & $3.58 \pm 0.12$ & $3.93 \pm 0.39$ & $1.66 \pm 0.11$ \\
\hline Wc \% & $3.21 \pm 0.20$ & $3.59 \pm 0.36$ & $1.60 \pm 0.09$ \\
\hline
\end{tabular}

Tabla 2. Valores de densidad aparente (Da), densidad real (Dr), porosidad accesible al agua (Pa), contenido de agua en saturación (Ws), por absorción libre (Wa) y por succión capilar (Wc). Valores medios \pm desviación estándar para cinco probetas por cada muestra.

La calidad de una roca como material constructivo y su susceptibilidad frente a los factores de alteración vienen determinados más que por su composición química y mineralógica por sus propiedades físicas y fundamentalmente por su sistema poroso (BENAVENTE et al., 2004). La cantidad de huecos de una roca y el grado de conexión entre ellos son fundamentales pues de estas características depende su comportamiento frente al agua, principal agente de alteración (WILSON, 2004). Como se observa en la Tabla 2, todas las muestras analizadas presentan una porosidad abierta $(\mathrm{Pa})$ muy elevada especialmente los bloques 2 y 3 siendo considerablemente menor en el bloque 4 . Los elevados valores de $\mathrm{Pa}$ concuerdan con lo observado en el estudio petrográfico, en el que se puso en evidencia la existencia de una densa red de fisuras intra, inter y transgranulares. Estos resultados indican que el granito de Paredes ha sufrido una alteración importante desde el punto de vista físico. A este respecto hay que recordar que los granitos sanos que se comercializan como rocas ornamentales tienen una porosidad abierta 
entre $0,1 \%$ y $2 \%$, con frecuencia en torno al 1\% (UNE-EN, 1936-1999).

Una porosidad abierta elevada implica una gran capacidad de absorber agua por diferentes mecanismos. Los valores del contenido de agua que la roca puede absorber libremente por inmersión (Wa), por capilaridad (Wc) y forzando su entrada a vacío (Ws) son muy similares, lo que indica que los huecos de la roca están bien comunicados entre sí y con el exterior, de ahí que la roca absorberá el agua con rapidez pero también se secará con facilidad. Esta característica resulta favorable pues determina que el tiempo de residencia de las disoluciones alterantes en el interior de la piedra será corto. Esto siempre que no haya otras circunstancias que ralenticen el secado, como por ejemplo un deficiente drenaje o la cobertura biológica que impida la evaporación.

\section{RESUMEN Y CONCLUSIONES}

Todos los resultados indican que el granito de Paredes se encuentra notablemente alterado. En primer lugar, rasgos como la extinción ondulante del cuarzo y la cloritización y plegamiento de las biotitas, indican que ha experimentado una alteración premeteórica y procesos de deformación tectónica, antes incluso de aflorar a la superficie. Estos fenómenos han sido descritos por numerosas autores en los granitos hercínicos de Galicia (e.g., CAPDEVILA y FLOOR, 1970; BELLIDO et al., 1987).

La consecuencia más importante de estos fenómenos es una intensa fisuración de la roca a todos los niveles, macro y microscópico. En campo es notoria la densa red de diaclasas sin embargo el estudio específico realizado acerca de sus características y de la estabilidad geomecánica de los afloramien- tos, revela que esto no supone un riesgo de pérdida masiva de roca especialmente en los planos que poseen grabados. Sin embargo se constató la clara influencia de las diaclasas en la canalización y penetración del agua en la masa rocosa facilitando el proceso de meteorización.

La dinámica de oxidación-reducción del hierro ha sido sin duda un mecanismo activo en la meteorización del granito de Paredes. El hierro presente en la biotita se encuentra en estado reducido $(\mathrm{Fe}++)$ que en contacto con las aguas superficiales cargadas de oxígeno se oxida formando hidróxidos y oxihidróxidos que salen de la estructura del mineral y pasan a recubrir y rellenar las fisuras de los minerales de alrededor confiriendo a la roca una coloración característica. Como se ha comentado, es frecuente en muchos afloramientos la presencia de una zona superficial decolorada por debajo de la cual se observa una acumulación de oxihidróxidos. La decoloración implica la solubilización y lavado del hierro lo que requiere condiciones reductoras que pueden darse cuando se produce un estancamiento de agua. La acidez y la presencia de materia orgánica en el medio (debido a los componentes orgánicos de los suelos) facilitarían el proceso. Esto conduce a pensar que tal vez estas alteraciones se produjeron estando los afloramientos enterrados o parcialmente enterrados. Hay que tener en cuenta que la dinámica de erosión-acumulación fue muy activa en esta área y las superficies que ahora están al aire pudieron estar bajo tierra en otra época (COSTA-CASAIS et al., 2009).

El agua es sin duda el principal agente de alteración. Es también responsable de los procesos de hidrólisis que afectan a la biotita que se transforma en vermiculita hidroxialumínica, y a los feldespatos sobre 
todo a las plagioclasas, cuyo producto de meteorización más común es la caolinita. Estos minera-les neoformados son arcillas y su presencia en los granitos aunque sea en una proporción mínima supone una merma muy importante de su calidad y un problema grave para la conservación de los grabados pues disminuye la dureza de la piedra y su resistencia a la abrasión. Así, la forma de deterioro más generalizada y preocupante es la desagregación arenosa o arenización, esto es la pérdida de masa rocosa grano a grano.

En conclusión, el granito de Paredes presenta un importante grado de alteración pero es probable que ya fuera así cuando los grabados fueron hechos. Hay que pensar que su homogeneidad, su granulometría bastante fina y su notable alteración, que hace que sea más blando, son características que facilitan la labor del grabado. Quizás esta mala calidad de la piedra que la hace idónea para grabar es la razón de la extraordinaria riqueza de petroglifos de esta área.

Desde el punto de vista de la conservación las recomendaciones más importantes que se pueden hacer son, por una parte, canalizar adecuadamente las escorrentías superficiales en el entorno de los petroglifos y favorecer un buen drenaje de los mismos para evitar que el agua se acumule sobre ellos o a su alrededor. Es evidente que el agua ha sido y sigue siendo el principal agente de deterioro pues interviene en las reacciones químicas de meteorización, disminuye la resistencia mecánica de la roca y favorece la colonización biológica. Por otra parte, es de la máxima importancia evitar que los visitantes pisen o manipulen los grabados puesto que la piedra está en un estado muy vulnerable frente a la erosión.

\section{AGRADECIMIENTOS}

Este estudio fue financiado por la Xunta de Galicia mediante el proyecto PGIDIT 05CCP20301PR coordinado por la Prof. Benita Silva. Las autoras agradecen además la ayuda recibida como Grupo de Referencia Competitiva (GRC) para el periodo 20192021 (ED431C 2018/32).

\section{REFERENCIAS}

BELLIDO MULAS, F., GONZÁLEZ LODEIRO, F., KLEIN, E., MARTÍNEZ CATALÁN, J.R., PABLO MACÍA, J.G. (1987). Las rocas graníticas hercínicas del Norte de Galicia y occidente de Asturias. Colección Memorias. Tomo 101. IGME.

BENAVENTE, D., GARCÍA DEL CURA, M. A., FORT, R., ORDÓÑEZ, S. (2004). Durability estimation of porous building stones from pore structure and strength. Engineering Geology 74 (1), 113-127.

CAPDEVILA, R., FLOOR, P. (1970). Les different types de granites hercyniens et leur distribution dans le nor-ouest de Espagne [The different types of Hercynian granites and their distribution in the north-west of Spain]. Boletín Geológico y Minero, 81, 215-225.

COSTA-CASAIS, M., MARTÍNEZCORTIZAS, A., PONTEVEDRA-POMBAL, X., CRIADO-BOADO, F. (2009). Analysis of landforms in geoarchaeology: Campo Lameiro, NW Iberian Peninsula. Memorie Descrittive della Carta Geologica d'Italia LXXXVII: 39-52.

GRANT, W.H. (1969). Abrasion pH, an index of chemical weathering. Clays and Clay Minerals, 17, 151-155. 
R.I.L.E.M. (1980). Essais recommandés pour mesurer l'altération des pierres et évaluer l'eficacité des méthodes de traitements. Matériaux et Constructions, Bull. RILEM, 13 (75), pp. 216-220.

ROMERO, R., TABOADA, T., GARCÍA, C., MACÍAS, F. (1987). Utilización del pH de abrasión como un índice del grado de evolución de la alteración y edafogénesis en suelos graníticos de la provincial de La Coruña. Cadernos do Laboratorio Xeolóxico de Laxe, 11, 171-182.
UNE-EN 1936:1999. Natural stone test methods, Determination of real density and apparent density, and of total and open porosity, AENOR (Spanish Association for Standardisation and Certication).

WILSON, M. J. (2004). Weathering of primary rock forming minerals: processes, products, and rates. Clay Minerals, 39, 233-266. 